\title{
INCIDENCIA DE INFECCIÓN DEL SITIO QUIRÚRGICO EN EL SERVICIO DE CIRUGİA I, HOSPITAL DR. MIGUEL PÉREZ CARREÑO. 2019-2021. ESTUDIO OBSERVACIONAL
}

\author{
SILVIA PIÑANGO $1^{*}$ (D) \\ LUIS LEVEL ${ }^{2}$ (D) \\ CRISTINA INCHAUSTI ${ }^{3}$ \\ SURGICAL SITE INFECTION INCIDENCE IN SURGERY \\ SERVICE I, HOSPITAL DR. MIGUEL PÉREZ CARREÑO. \\ 2019-2021. OBSERVATIONAL STUDY
}

\section{RESUMEN}

La infección del sitio quirúrgico es uno de los tipos más frecuentes de infección asociada al cuidado de la salud, tiene un impacto en el estado físico y mental de los pacientes. aumentando el riesgo de mortalidad. El objetivo de este estudio es determinar la incidencia de infección del sitio quirúrgico en cirugías electivas y de emergencia, y su distribución según la patología. Métodos: Estudio retrospectivo y observacional en el cual se incluyeron pacientes del servicio de Cirugía General I del Hospital Miguel Pérez Carreño, entre enero de 2019 y julio de 2021, a quienes se les realizó un procedimiento quirúrgico. Resultados: Se incluyeron 1341 pacientes. La incidencia global de infección de sitio quirúrgico fue de 9,77\%, la mayoría de las infecciones fueron en cirugías de emergencia con una incidencia de 10,7\% y de 4,17\% en cirugías electivas. Las patologías con mayor porcentaje de infección fueron el trauma abdominal, seguido de la obstrucción intestinal. La incidencia de infección del sitio quirúrgico en patología apendicular fue de $10,4 \%$. El microorganismo aislado con mayor frecuencia en Ios cultivos realizados fue E. coli. Conclusiones: La infección del sitio quirúrgico es una complicación frecuente en la cirugía de emergencia y requiere ser considerada y prevenida en el trauma abdominal, las obstrucciones intestinales y las peritonitis apendiculares. Conocer la incidencia de está complicación y comparar con estudios internacionales es fundamental para establecer protocolos propios para su control y prevención

Palabras clave: Infección del sitio quirúrgico, trauma abdominal, apendicitis aguda, patología biliar, obstrucción intestinal, profilaxis antibiótica

\footnotetext{
1. Cirujano General, Adjunto Servicio Cirugía General, Hospital “Dr. Miguel Pérez Carreño”. IVSS. Caracas-Venezuela.

2. Cirujano General. Jefe de Servicio de Cirugía I. Director del Postgrado de Cirugía General de la Universidad Central de Venezuela. Hospital Dr. Miguel Pérez Carreño. Caracas Venezuela. Correo-e: levelluis@yahoo.com

3. Cirujano General. Hospital Dr. Miguel Pérez Carreño. Caracas- Venezuela
}

\begin{abstract}
Surgical site infection is one of the most frequently reported types of healthcare-associated infections, it has a negative impact on patients physical and mental health and increase the risk of mortality. The objective of this study is to determine the incidence of surgical site infection in elective and emergency surgeries, and its distribution according to the pathology. Methods: Retrospective and observational study, in which patients from the General Surgery Service I of the Hospital Miguel Pérez Carreño were included, between January 2019 and July 2021, who went under a surgical procedure. Results: In total 1341 patients were included. The overall incidence of surgical site infection was $9.77 \%$, most of the infections were in emergency surgery with an incidence of $10.7 \%$ and $4.17 \%$ in elective surgeries. The pathologies with the highest percentage of infection were abdominal trauma followed by bowel obstruction. The incidence of surgical site infection in appendicular pathology was 10.4\%. The most frequently isolated pathogens in the cultures performed was E.coli. Conclusions: Surgical site infection is a frequent complication in emergency surgery and needs to be considered and prevented in abdominal trauma, bowel obstructions, and complicated appendicitis. Knowledge of the incidence of this complication and comparing it with international studies is essential to establish own protocols for its control and prevention.
\end{abstract}

Key words: Surgical site infection, abdominal trauma, acute appendicitis, biliary pathology, intestinal obstruction, antibiotic prophylaxis
Recepción: 14/08/2021

Aprobación: 24/10/2021

DOI: $10.48104 /$ RVC.2021.74.2.6

muw.revistavenezolanadecirugia.com

* Aunque el autor principal es el editor de la revista, esta artículo fue sometido a arbitraje doble ciego al igual que los demás artículos de investigacion 


\section{INTRODUCCIÓN}

Las infecciones del sitio quirúrgico (ISO) son uno de los tipos más frecuentes de infecciones asociadas al cuidado de salud, representando el 20\% de las mismas. El centro de prevención y control de enfermedades (CDC) define ISO como una infección en la parte del cuerpo donde tuvo lugar la cirugía, que ocurre dentro de los primeros 30 días de un procedimiento quirúrgico o en el primer año en procedimientos con uso de material protésico. ${ }^{(1,2)}$

Este tipo de complicación tiene un impacto negativo en el estado físico y mental del paciente. Es responsable del aumento de la estancia hospitalaria, reingresos en el postoperatorio, ingresos a las unidades de cuidados intensivos, costos significativamente mayores y retraso del inicio de terapias adyuvantes. ${ }^{\text {(3) }}$ Se ha descrito un aumento del riesgo de mortalidad y a pesar de que la mayoría de los pacientes se recuperan sin secuelas, el 77\% de la mortalidad de los pacientes con ISO se puede atribuir a la infección. ${ }^{12}$ )

La identificación, el control y la prevención de las infecciones es un problema institucional, que requiere la interacción de los diferentes factores del sistema de atención de salud, a través de los Comité de infecciones de cada institución. (4)

El CDC describe tres niveles de ISO: ${ }^{(3)}$

1. Incisional superficial: Afecta piel y tejido celular subcutáneo

2. Incisional profunda: Afecta la fascia y la capa muscular

3. Infección de órgano-espacio: Involucra alguna parte de la anatomía diferente a la incisión que ha sido abierta o manipulada durante el procedimiento quirúrgico como el peritoneo.

La prevención de la ISQ consta de tres aspectos fundamentales la profilaxis antimicrobiana, preparación de la piel y la normotermia preoperatoria. ${ }^{(4,5)}$

El principal beneficio del antibiótico preoperatorio es la reducción del inóculo viable en la herida durante la incisión quirúrgica. La indicación de profilaxis y la elección del antibiótico debe depender del procedimiento, tipo de herida, riesgo de infección postoperatoria y patógenos con mayor probabilidad de causar ISO. Debe administrarse entre 30 minutos a una hora antes de la incisión, o en las dos horas previas para vancomicina o ciprofloxacina. La dosis debe ajustarse al peso. El antibiótico no debe producir efectos adversos y no debe tener consecuencias desfavorables para la microflora de los pacientes del hospital y de la comunidad. ${ }^{(2,6)}$

En heridas limpias no está indicado el uso sistemático de profilaxis antibiótica, se reserva solo para el uso de material protésico. Las heridas limpias contaminadas y contaminadas deben recibir siempre antibioticoterapia profiláctica. Las heridas sucias o con infecciones establecidas deben recibir profilaxis para evitar la diseminación de la infección, adicional al inicio temprano del tratamiento para la infección que ya está establecida. (4)

El objetivo del presente estudio es determinar la incidencia de infección del sitio quirúrgico durante los últimos 30 meses en el Servicio de Cirugía I del Hospital Dr. Miguel Pérez Carreño y comparar con estudios previos nacionales e internacionales.

\section{MÉTODOS}

Se trata de estudio de cohorte, retrospectivo, descriptivo, observacional realizado en el Servicio de Cirugía I del Hospital Dr. Miguel Pérez Carreño en Caracas, Venezuela, entre enero de 2019 a julio de 2021 . Fueron incluidos todos los pacientes a quienes se les realizó una intervención quirúrgica de emergencia o electiva.

Se utilizó antibioticoterapia profiláctica en los 30 minutos previos a la intervención quirúrgica, en heridas limpias con uso de material protésico, limpias contaminadas y contaminadas. Se indicó tratamiento antibiótico postoperatorio en heridas sucias o con infecciones establecidas. La cobertura antibiótica se realizó según los gérmenes más frecuentemente identificados en la literatura para la patología y el órgano afectado. ${ }^{(4)}$

Se realizó antisepsia de la piel previo acto quirúrgico con antisépticos en base a yodo. No se utilizaron métodos específicos para mantener normotermia preoperatoria e intraoperatoria por no contar con dispositivos necesarios en la institución.

Se determinó ISO por la aparición de signos de inflamación, drenaje purulento, presencia de colecciones intrabdominales y sepsis.

Este estudio fue realizado según las recomendaciones y directrices para la presentación de informes de estudios observacionales del grupo STROBE. ${ }^{(7)}$

Se calculó frecuencia y porcentajes en las variables nominales y la comparación de estas se realizó mediante la prueba chicuadrado. Se consideró un valor estadísticamente significativo si el valor de $\mathrm{p}<0,05$. Los datos fueron tabulados y procesados con Epi info7®.

\section{RESULTADOS}

Se incluyeron 1341 pacientes con procedimientos quirúrgicos dentro de los cuales el 85,7\% fueron cirugías de emergencia y el restante procedimientos quirúrgicos programados o cirugías electivas.

Durante el período estudiado se diagnosticaron 131 ISO representando un 9,77\% del global de pacientes. La incidencia de ISO en cirugías de emergencia fue de 10,7\% y de 4,17\% en cirugías electivas. (Figura 1)

La presentación de ISO según las patologías se describe en la Figura 2.

\section{Patología apendicular}

Se realizaron 326 apendicectomías por apendicitis aguda de las cuales el 83,44\% presentó solo afectación local del peritoneo. El 32\% de los casos se abordó vía laparoscópica.

La incidencia general de ISO fue de 10,42\% y de estas el 61,76\% fueron pacientes que presentaban peritonitis difusa al 


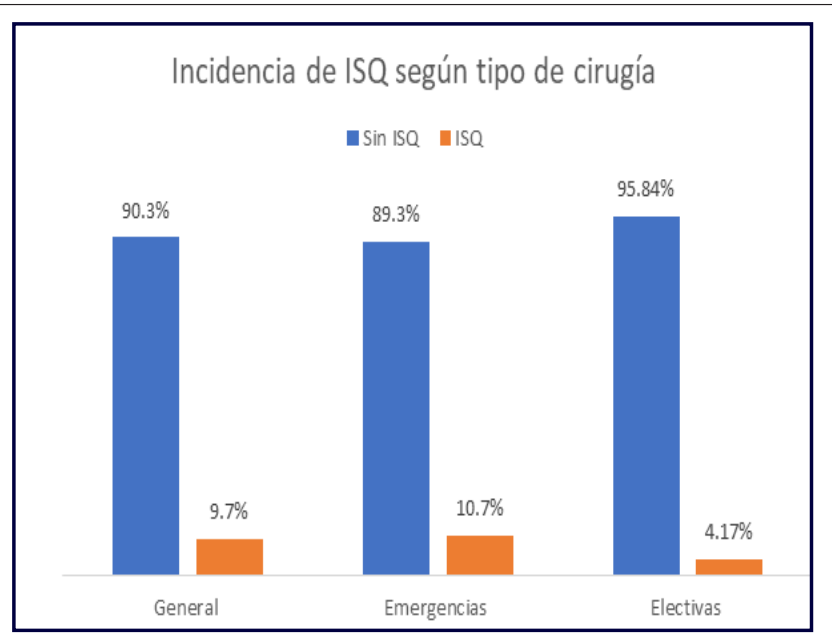

Figura 1. Incidencia de ISO general y según tipo de cirugía

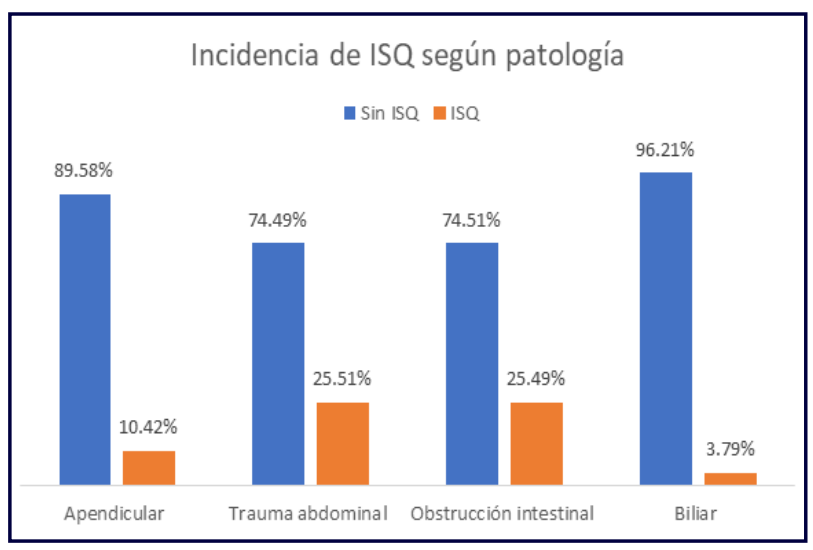

Figura 2. Incidencia de ISQ según patología

momento de la intervención.

La incidencia de ISO en el abordaje convencional fue de 11,71\% vs 7,69\% en cirugías laparoscópica. $p=0,26$.

ISO en patologías apendicular según grado de afectación peritoneal y abordaje: (Tabla 1)

Afección local: La incidencia de ISO fue de 5,85\%. En cirugías laparoscópica 3,19\% vs 5,62\% en cirugía convencional. $p=0.37$

Peritonitis difusa: Incidencia de ISO 38,88\%. En cirugías laparoscópicas 50\% vs 36,36\% en cirugía convencional. $p=0,42$

Obstrucción intestinal: Se realizaron 102 cirugías en pacientes con diagnóstico de obstrucción intestinal de los cuales el 25,49\% presentó infección de sitio quirúrgico.

Trauma abdominal: Se realizaron 98 cirugías en pacientes tanto con trauma abdominal cerrado como con trauma abdominal penetrante, la incidencia de ISO fue de 25,5\%.

Patología biliar: Se realizó cirugía biliar en 158 pacientes, el 3,79\% presentó ISO.

En relación a la identificación del microorganismo involucrado tenemos que de las 131 infecciones detectadas se realizó cultivo en el 36,64\% de los casos. El principal microorganismo aislado fue Escherichia coli en un 47,91\% de los cultivos informados.

\section{DIsCuSIÓN}

La Sociedad Americana de Cirujanos estima 2 al $5 \%$ de infección de sitio quirúrgico global,(2) sin embargo, la incidencia de ISO es mayor en las cirugías abdominales en comparación a otro tipo de cirugías; informan una incidencia de 15 al 25\% de ISO dependiendo del grado de contaminación.(8) De Simone y colaboradores describen hasta un 14\% de ISO después de cualquier tipo de cirugía abdominal, lo cual corresponde con los datos obtenidos en este estudio. Los resultados también fueron similares a los reportados en el año 2015 en el mismo servicio del Hospital Miguel Pérez Carreño, en el cual se describe una incidencia de 150 de 10,31\%. ${ }^{|3,9|}$

La incidencia de ISO varía según el tipo de cirugías desarrolladas en el centro, de este modo se describe que instituciones donde se realiza cirugías de emergencia, cirugía de trauma, cirugía colorectal o cirugías oncológicas tienen diferente frecuencia de $15 \mathrm{SO}{ }^{(3)}$

La cirugía de emergencia es un factor de riesgo de ISO por factores como mayor grado de contaminación de las heridas, duración prolongada de la cirugía, comorbilidades del paciente, entre otros. ${ }^{(3)}$ Esto se refleja en el porcentaje de ISO de cirugías de emergencia del presente estudio, este porcentaje fue menor en comparación al estudio realizado en 2015 en el mismo servicio donde se evidencia 12,95\% de ISO, sin embargo, en cirugías electiva el porcentaje fue mayor 4,17\% vs 3,92\% en el estudio previo. ${ }^{(9)}$ Es importante hacer notar que durante este periodo las cirugías electivas en general estaban constituidas por cirugías de emergencia que habían sido diferidas en espera de completar estudios diagnósticos, esperar turno quirúrgico oportuno, etc., en vista de la alta demanda de quirófanos y la escasa disponibilidad de los mismos.

La incidencia de ISO post apendicectomía global fue mayor a lo descrito por Danwang y colaboradores quienes obtuvieron una incidencia general de 7\%. La incidencia puede variar según la región, desde 5\% en Europa hasta un 12,6\% en África. (10)

Se describe una incidencia general $11 \%$ en apendicectomías abiertas y de 4,6\% en apendicectomías laparoscópicas. Al comparar los tipos de abordaje no hubo diferencia estadísticamente significativa, sin embargo debemos considerar que el tamaño de la muestra interfiere con el grado de significancia. ${ }^{(10)}$ De igual manera es importante hacer mención a la alta incidencia de peritonitis apendiculares intervenidas en virtud de retrasos en acudir a consulta a nuestra emergencia o a retraso en la asignación de turnos quirúrgicos una vez hospitalizados en la institución.

Las ISO post colecistectomía es mayor en colecistectomía abierta que en colecistectomía laparoscópica se informa del 1,1\% hasta el 8,4\% vs 0,3\% hasta 3,4\% respectivamente. En España se informa un 3,6\% de ISO general post colecistectomía. . $^{11,121}$ 
Tabla 1. Incidencia de ISQ en patología apendicular. Según abordaje y afectación peritoneal

\begin{tabular}{lcccccc}
\hline & \multicolumn{2}{c}{$\begin{array}{c}\text { Laparoscopia } \\
(\mathbf{n = 1 0 4 )}\end{array}$} & $\begin{array}{c}\text { Convencional } \\
(\mathbf{n = 2 2 2})\end{array}$ & \\
\hline & No. & $\%$ & No. & $\%$ & $x^{2}$ & $\mathrm{P}$ \\
ISO & 8 & 7,69 & 26 & 11,71 & & \\
$\mathrm{Si}$ & 96 & 92.31 & 196 & 88.29 & 1,22 & 0,26 \\
$\mathrm{No}$ & & & & &
\end{tabular}

Afectación peritoneal

\begin{tabular}{lcccccc} 
Localizada & \multicolumn{5}{c}{ Laparoscopia $(\mathrm{n}=94)$} & \multicolumn{2}{c}{ Convencional $(\mathrm{n}=178)$} & \\
\cline { 2 - 4 } ISO & 3 & 3,19 & 10 & 5,62 & 0,79 & 0,37 \\
Sin ISO & 91 & 96,81 & 168 & 94,38 & & \\
Peritonitis Difusa & \multicolumn{2}{c}{ Laparoscopia $(\mathrm{n}=10)$} & Convencional $(\mathrm{n}=44)$ & & \\
ISO & 5 & 50 & 16 & 36,36 & 0,63 & 0,42 \\
Sin ISO & 5 & 50 & 28 & 63,64 & & \\
\hline
\end{tabular}

Estos datos son similares a los obtenidos en nuestro estudio. Es importante destacar que las colecistectomías electivas representan en muchos casos colecistectomías de emergencia que han sido diferidas y pasan a tener períodos de hospitalización mayores a 72 horas antes de la cirugía, lo cual predispone al paciente a colonización por microorganismos nosocominales o gérmenes resistentes.

El porcentaje de ISO en pacientes con diagnóstico de obstrucción intestinal fue alto. Esta patología es un factor de riesgo para ISO ya existe una alteración de la barrera intestinal, debido al ayuno prolongado y la dilatación de la luz intestinal, independientemente de si hay apertura del tracto gastrointestinal, condicionando la traslocación bacteriana.(13) El retraso en el manejo quirúrgico de los pacientes con obstrucciones intestinales constituye un factor agravante que predispone a la aparición de complicaciones e ISO.

El porcentaje de ISO en pacientes con trauma abdominal también fue alto, mayor al reportado en el mismo centro en el año 2014 de 16\%.(14) En vista de este resultado se recomienda realizar un estudio de los factores de riesgos y áreas que se deben mejorar en pacientes con esta patología.

La realización del cultivo es un elemento fundamental en el manejo de los pacientes con infecciones, permite adecuar el tratamiento haciéndolo específico para el paciente y el microorganismo involucrado, disminuyendo los efectos adversos del uso indiscriminado de antibióticos. ${ }^{(6,15)}$ Sin embargo, en nuestro centro se logró realizar cultivo en menos de la mitad de las infecciones detectadas.

El microorganismo aislado con más frecuencia, en casi la mitad de los cultivos fue Escherichiacoli, Io cual corresponde con estudios previos realizados en el mismo hospital e informes internacionales, en los cuales se describe la prevalencia de gérmenes gram negativos, específicamente E. coli en cirugías abdominales. ${ }^{(16,17)}$

La evidencia actual sugiere que la antisepsia de la piel con preparación a base de alcohol es más efectiva que otras soluciones acuosas y se debe utilizar a menos que exista alguna contraindicación. El alcohol presenta un efecto bactericida rápido, sin embargo, no tiene efecto antibacteriano persistente, por lo que se recomienda utilizar alcohol con yodo o clorhexidina para actividad bactericida prolongada. (18) En este estudio se realizó antisepsia solo con soluciones a base de yodo.

Durante el año 2020 hubo una disminución de los procedimientos quirúrgicos laparoscópicos en el hospital Pérez Carreño, dado que al inicio de la pandemia de la enfermedad viral por coronavirus no estaba demostrado la seguridad de este abordaje quirúrgico ${ }^{(19)}$. Este hecho pudo aumentar la incidencia de ISO en ese período de tiempo, se describe una disminución significativa de ISO en cirugías laparoscópicas en comparación con cirugías abiertas. ${ }^{(13)}$

De este modo podemos concluir que la incidencia de 150 durante este período fue alta, aunque está en relación con la incidencia reportada en otros centros y el tipo de patología manejada. El déficit de antisepsia a base de sustancias alcoholadas, el retraso en la intervención quirúrgica, el tipo de patología abordada, el tiempo de hospitalización preoperatorio, la ausencia de medidas para mantener la normotermia del paciente durante el acto operatorio y el postoperatorio, constituyen algunos de los elementos que deben ser controlados para disminuir el porcentaje de infecciones. El uso de antibiótico indiscriminado no sustituye el cumplimiento adecuado de las otras medidas para el control de infecciones.

Se requieren futuros estudios prospectivos y multicéntricos para el conocimiento de índices nacionales de infecciones según patología, además de establecer los factores de riesgo para las ISO en los procedimientos quirúrgicos por trauma abdominal y obstrucción intestinal para buscar disminuir el porcentaje de ISO en estas patologías.

\section{Aprobación Ética}

Para este tipo de estudio no se requiere consentimiento formal. 


\section{Conflicto de InTEReses}

Los autores declaran no tener conflicto de interés sobre el presente estudio.

\section{REFERENCIAS}

1. Badia JM, Casey AL, Petrosillo N, Hudson PM, Mitchell SA, Crosby C. Impact of surgical site infection on healthcare costs and patient outcomes: a systematic review in six European countries. Vol. 96, J Hosp Infec. W.B. Saunders Ltd; 2017. 1-15. https://wnw. journalofhospitalinfection.com/article/S0 $195-6701$ (17)30 135-4/ fulltext

2. Ban KA, Minei JP, Laronga C, Harbrecht BG, Jensen EH, Fry DE, et al. American College of Surgeons and Surgical Infection Society: Surgical Site Infection Guidelines, 2016 Update. J of Am Coll Surg. 2017 Jan 1:224(1):59-74.http://www.journalacs.org/article/ S1072751516315630/fulltext

3. Belinda DS, Massimo S, Federico C, Chad B, Pietro B, Massimo C, etal. Intraoperative surgical site infection control and prevention: a position paper and future addendum to WSES intra-abdominal infections guidelines. World J of Emer surg: WJES. 2020 Feb 10;15(1). https:// pubmed.ncbi.nlm.nih.gov/32041636/

4. Blanco L, Castillo Y, Cebrián J, Hartmann C, Marín I, Ottolino P, etal. Manual Consenso Venezolano de Infecciones Quirúrgicas. Rev Venez Cir. 20 17. https://sociedadvenezolanadecirugia.com/wp-content/ uploads/20 19/09/Manual-Infecciones-Quir\%C3\%BArgicas.pdf

5. Berriós-Torres SI, Umscheid CA, Bratzler DW, Leas B, Stone EC, Kelz RR, etal. Centers for disease control and prevention guideline for the prevention of surgical site infection, 2017. JAMA Surgery. 2017 Aug 1;152(8):784-91. https://jamanetwork.com/journals/jamasurgery/ fullarticle/2623725

6. Piñango S, Marín I. Profilaxis quirúrgica y antibioticoterapia inicial. Rev Venez Cir. 20 18;7 1 11). https://muw.revistavenezolanadecirugia. com/index.php/revista/article/view/31

7. Von Elm E, Altman DG, Egger M, Pocock SJ, Gøtzsche PC, Vandenbroucke JP. The Strengthening the Reporting of Observational Studies in Epidemiology (STROBE) statement: Guidelines for reporting observational studies. Ann Intern Med. 2007 Oct 16;147(8):573-7. https://pubmed.ncbi.nlm.nih.gov/17938396/

8. Alkaaki A, Al-Radi OO, Khoja A, Alnawawi A, Alnawawi A, Maghrabi A, et al. Surgical site infection following abdominal surgery: A prospective cohort study. Can J Surg. 2019 Apr 1;62(2):111-7. https://pubmed. ncbi.nlm.nih.gov/30907567/

9. Cebrian J, Ottolino P. Epidemiología y definiciones en infecciones quirúrgicas. Rev Ven Cir. 2017:70(1). https://mww. revistavenezolanadecirugia.com/index.php/revista/article/view/ 148

10. Danwang C, Bigna JJ, Tochie JN, Mbonda A, Mbanga CM, Nzalie RNT, etal. Global incidence of surgical site infection after appendectomy: A systematic review and meta-analysis. Vol. 10, BMJ Open; 2020. https://pubmed.ncbi.nlm.nih.gov/32075838/

11. Warren DK, Nickel KB, Wallace AE, Mines D, Tian F, Symons WJ, et al. Risk factors for surgical site infection after cholecystectomy. Open Forum Infect Dis. 2017 Mar 1;4(2). https://pubmed.ncbi.nlm.nih. gov/28491887/

12. Rodriguez-Caravaca G, Gil-Yonte P, Del-Moral-luque JA, Lucas WC, Fernández-Cebrián JM, Durán-Poveda M. Rates of surgical site infection in cholecystectomy: Comparison between a University Teaching hospital, Madrid Region, Spain, and USA rates. Rev Invest Clin. 2017 Nov 1;69(6):336-43. https://mww.medigraphic.com/ cgi-bin/new/resumen.cgi?IDARTICULO=78180

13. Li Z, Li H, Lv P, Peng X, Wu C, Ren J, Wang P. Prospective multicenter study on the incidence of surgical site infection after emergency abdominal surgery in China. Sci Rep. 2021 Apr 8;1 1 (1):7794. doi: 10.1038/s4 1598-021-87392-8. https://pubmed.ncbi.nlm.nih. gov/33833359/

14. Piñango S, Level L, Hernández F, Rojas A. Antibiotico en trauma abdominal penetrante 7-3 días. Revista Centro Médico CaracasVenezuela. 2014;141(2):6-11. https://mww.revistacentromedico. org/ediciones/2014/2/art-3/

15. Castillo Y, Piñango S. Tratamiento antimicrobiano específico de las infecciones quirúrgicas. Rev Venez Cir. 2018;71(1). https://wnw revistavenezolanadecirugia.com/index.php/revista/article/view/32

16. Zhang XF, Chen J, Wang PG, Luo SM, Liu NX, Li XM, et al. Surgical site infection after abdominal surgery in China: a multicenter cross-sectional study. Zhonghua Wei Chang Wai Ke Za Zhi. 2020 Nov 25:23(11):1036-1042. Chinese. doi: 10.3760/ cma.j.cn.441530-20200810-00470. PMID: 33212551. https:// pubmed.ncbi.nlm.nih.gov/33212551/

17. El-Kholy AA, Elanany MG, Sherif MM, Gad MA. High Prevalence of VIM, KPC, and NDM Expression among Surgical Site Infection Pathogens in Patients Having Emergency Surgery. Surg Infect. 2018 Aug 1;19(6):629-33. https://pubmed.ncbi.nlm.nih.gov/29979638/

18. Ling ML, Apisarnthanarak A, Abbas A, Morikane K, Lee KY, Warrier $A$, et al. APSIC guidelines for the prevention of surgical site infections. Antimicrob Resist Infect Control. 2019 Nov 12;8(1). https://muw. ncbi nlm.nih.gov/pmc/articles/PMC6852795/

19. Adrianus R, Burger NB, Ceccaroni M, Zhang J, Tuynman J, Mabrouk M, etal. COVID-19 and Laparoscopic Surgery: Scoping Review of Current Literature and Local Expertise. JMIR Public Health Surveil 2020;6(2) doi:10.2196/18928 https://pubmed.ncbi.nlm.nih. gov/32406853/ 